\title{
Inhaltsverzeichnis
}

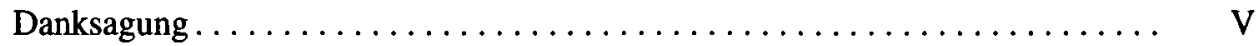

Vorwort $\ldots \ldots \ldots \ldots \ldots \ldots \ldots \ldots \ldots \ldots \ldots \ldots \ldots \ldots \ldots \ldots \ldots \ldots \ldots \ldots$

Teil I

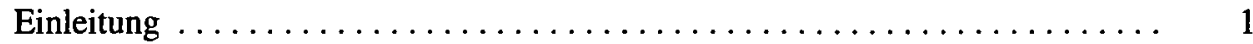

1 Anliegen und Aufbau des Buches . . . . . . . . . . . . . . . 3

Teil II

Perspektiven zum Planungsproze $\mathbb{B} \ldots \ldots \ldots \ldots \ldots \ldots \ldots \ldots \ldots \ldots$

2 Der Streit zwischen Planern und Inkrementalisten ............... 11

Mintzberg, H. „The Design School: Reconsidering the Basic Premises of Strategic

Management", Strategic Management Journal, Jg. 11, 1990, S. 171-195. . . . . . . . . . . 14

Ansoff, H. I. „Critique of Henry Mintzberg's ,The Design School: Reconsidering the

Basic Premises of Strategic Management ““, Strategic Management Journal, Jg. 12,

1991, S. 449-461. . . . . . . . . . . . . . . . . . . . . . . . . . . .

Weick, K. E. „Substitutes for Strategy“, in: Teece, D. (Hrsg.), The Competitive Challenge.

Strategies for Industrial Innovation and Renewal, Ballinger Publishing Company,

Cambridge, 1987, S. 221-233.

Teil III

Strategische Entscheidungsträger $\ldots \ldots \ldots \ldots \ldots \ldots \ldots \ldots \ldots \ldots \ldots$

3 Der Konflikt zwischen Prinzipalen und Agenten . . . . . . . . . . . 71

Tosi, H. L. und L. R. Gomez-Mejia. „The Decoupling of CEO Pay and Performance: An Agency Theory Perspective“, Administrative Science Quarterly, Jg. 34, 1989, S. 169-189. .

Zajac, E. J. „CEO Selection, Succession, Compensation and Firm Performance: A

Theoretical Integration and Empirical Analysis“, Strategic Management Journal, Jg. 11, 1990, S. 217-230. . . . . . . . . . . . . . . . . . . . . . . . . . . . . . . . . . . . . . . . .

4 Strategische Entscheidungsträger und Unternehmungsperformance . . . . . .

Hambrick, D. C. und P. A. Mason. „Upper Echelons: The Organization as a Reflection of Its Top Managers", Academy of Management Review, Jg. 9, 1984, S. 193-206. . . . . . . . .

Finkelstein, S. und D. C. Hambrick. „Top-Management-Team Tenure and Organizational Outcomes: The Moderating Role of Managerial Discretion", Administrative Science Quarterly, Jg. 35, 1990, S. 484-503. . . . . . . . . . . . . . . . . . . 
Thomas, J. B., Clark, S. M. und D. A. Gioia. „Strategic Sensemaking and Organizational Performance: Linkages Among Scanning, Interpretation, Action, and Outcomes", Academy of Management Journal, Jg. 36, 1993, S. 239-270 . . . . . . . . . . . . . . . . .

\section{Teil IV}

Externe Umweltanalysen

5 Branchenstruktur- und strategische Gruppenanalysen .............

Cool, K. O. und D. Schendel. „Strategic Group Formation and Performance: The Case of the U.S. Pharmaceutical Industry, 1963-1982“, Management Science, Jg. 33, 1987,

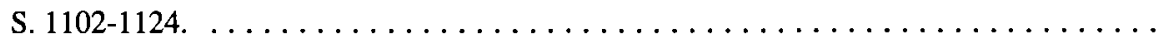
Reger, R. K. und A. S. Huff. „Strategic Groups: A Cognitive Perspective“, Strategic Management Journal, Jg. 14, 1993, S. 103-124 . . . . . . . . . . . . . . . . . . . .

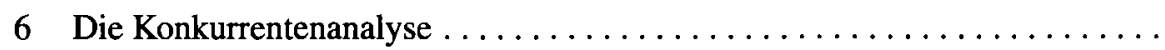

Weigelt, K. und I. MacMillan. „An Interactive Strategic Analysis Framework“, Strategic Management Journal, Jg. 9, Summer 1988 (Special Issue), S. 27-40 . . . . . . . . . . . . Zajac, E. J. und M. H. Bazerman. „Blind Spots in Industry and Competitor Analysis: Implications of Interfirm (Mis)perceptions for Strategic Decisions“, Academy of

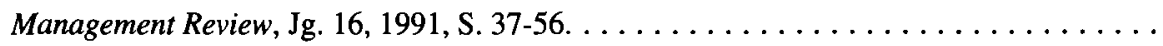
Jacobson, R. ,The ,Austrian' School of Strategy“, Academy of Management Review, Jg. 17, 1992, S. 782-807.

Teil V

Interne Umweltanalysen $\ldots \ldots \ldots \ldots \ldots \ldots \ldots \ldots \ldots \ldots \ldots \ldots \ldots \ldots$

7 Ressourcen und Fähigkeiten der Unternehmung $\ldots \ldots \ldots \ldots \ldots \ldots \ldots$

Peteraf, M. A. „The Comerstones of Competitive Advantage: A Resource-Based View“, Strategic Management Journal, Jg. 14, 1993, S. 179-191. . . . . . . . . . . . . . . Amit, R. und P. Schoemaker. „Strategic Assets and Organizational Rent", Strategic Management Journal, Jg. 14, 1993, S. 33-46.

\section{Teil VI}

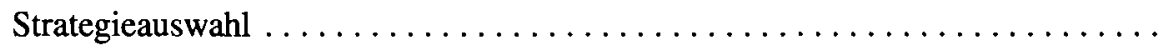

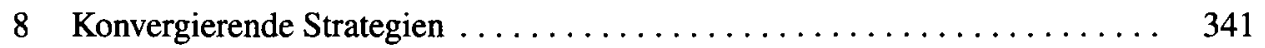

Haunschild, P. R. „Interorganizational Imitation: The Impact of Interlocks on Corporate Acquisition Activity“, Administrative Science Quarterly, Jg. 38, 1993, S. 564-592 . . . . . . Deephouse, D. L. „Does Isomorphism Legitimate?“, Academy of Management Joumal, Jg. 39, 1996, S. 1024-1039. . . . . . . . . . . . . . . . . . . . . . . . . . . . .

9 Gesamtunternehmungsstrategien $\ldots \ldots \ldots \ldots \ldots \ldots \ldots \ldots \ldots \ldots \ldots \ldots$

Hill, C. W. L. „Cooperation, Opportunism, and the Invisible Hand: Implications for Transaction Cost Theory“, Academy of Management Review, Jg. 15, 1990, S. 500-513 . . . 
Chatterjee, S. und B. Wernerfelt. „The Link Between Resources and Type of

Diversification: Theory and Evidence", Strategic Management Journal, Jg. 12, 1991,

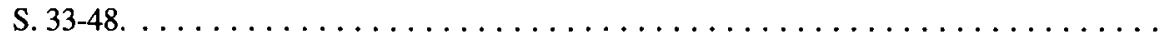

10 Geschäftsbereichsstrategien

Lieberman, M. B. und D. B. Montgomery. ,First-Mover Advantages“, Strategic

Management Journal, Jg. 9, Summer 1988 (Special Issue), S. 41-58. . . . . . . . . . . .

Hill, C. W. L. „Differentiation Versus Low Cost or Differentiation and Low Cost: A

Contingency Framework“, Academy of Management Review, Jg. 13, 1988, S. 401-412. . . .

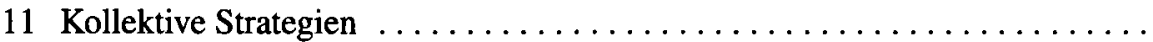

Bresser, R. K. F. „Kollektive Unternehmensstrategien“, Zeitschrift für Betriebswirtschaft,

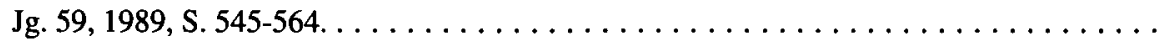

Eisenhardt, K. M. und C. B. Schoonhoven. ,Resource-based View of Strategic Alliance Formation: Strategic and Social Effects in Entrepreneurial Firms“, Organization Science, Jg. 7, 1996, S. 136-150.

\section{Teil VII}

Strategieimplementierung $\ldots \ldots \ldots \ldots \ldots \ldots \ldots \ldots \ldots \ldots \ldots$

12 Strukturen, Führungspersonal, Kulturen und Mikropolitik

Hill, C. W. L., Hitt, M. A. und R. E. Hoskisson. „Cooperative Versus Competitive Structures in Related and Unrelated Diversified Firms", Organization Science, Jg. 3, 1992, S. 501-521. Howell, J. M. und C. A. Higgins. „Champions of Technological Innovation“, Administrative Science Quarterly, Jg. 35, 1990, S. 317-341 . . . . . . . . . . . . . . . . .

\section{Teil VIII}

Strategische Kontrolle 579

13 Bewertung und Systeme

Goold, M. und J. J. Quinn. „The Paradox of Strategic Controls“, Strategic Management

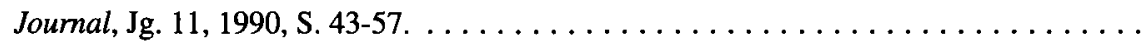

Eschen, E. „Finanzkennzahlen im Strategischen Management“, Arbeitspapier des

Lehrstuhls für Unternehmungsplanung und -politik, Freie Universität Berlin, 1998. . . . . .

Teil IX

Strategischer Wandel

14 Strategische Wahlfreiheit versus strukturelle Trägheit

Kelly, D. und T. L. Amburgey. „Organizational Inertia and Momentum: A Dynamic Model of Strategic Change", Academy of Management Journal, Jg. 34, 1991, S. 591-612 . . . . . . Hrebiniak, L. G. und W. F. Joyce. „Organizational Adaptation: Strategic Choice and Environmental Determinism“, Administrative Science Quarterly, Jg. 30, 1985, S. 336-349. 
Teil X

Epilog

673

15 Perspektiven für eine einheitliche Theorie des Strategischen Managements .. 675

Literaturverzeichnis.............................. 683

Stichwortverzeichnis $\ldots \ldots \ldots \ldots \ldots \ldots \ldots \ldots \ldots \ldots \ldots \ldots \ldots, 711$ 